\title{
Estimating the population of the Endangered flat-tailed tortoise Pyxis planicauda in the deciduous, dry forest of western Madagascar: a monitoring baseline
}

\author{
Richard P. Young, Anselme Toto Volahy, Robert Bourou \\ Richard Lewis, JoAnna Durbin and Julia E. Fa
}

\begin{abstract}
The Madagascar flat-tailed tortoise Pyxis planicauda is threatened with extinction through loss of its deciduous, dry forest habitat and illegal collection for the pet trade. Little is known of the population status of this species as no systematic survey across its range has previously been conducted. Surveying $P$. planicauda is problematic as it has cryptic colouration, is only active during the wet season, and spends periods of time hidden under leaf litter. In this study we designed a line transect survey, which incorporated an approximation of the amount of time tortoises were available for sampling, to produce an unbiased estimate of population size. We estimated density of $P$. planicauda in the main forest block in central Menabe, which represents the majority of its range, to be $0.40 \mathrm{ha}^{-1}$ with a total population size of nearly 28,000 individuals ( $95 \%$ confidence interval $16,323-47,669)$. This estimate is substantially higher than previous predictions of $<10,000$ individuals. Nevertheless, the species faces ongoing threats from deforestation and probably from illegal collection, although the recent designation of the Menabe forest as a protected area will probably help reduce these threats. This study provides a baseline density estimate of $P$. planicauda and a survey protocol for monitoring future population trends.
\end{abstract}

Keywords Distance sampling, dry forest, line transect survey, Madagascar, population monitoring, Pyxis planicauda, tortoise.

\section{Introduction}

7 he deciduous, dry forest of western Madagascar is one 1 of the world's biologically richest and most unique tropical dry forests (Guillaumet, 1984; Lowry et al., 1997). However, it has undergone a dramatic decline in extent and become highly fragmented through timber harvesting,

RichaRd P. Young (Corresponding author) and Julia E. FA Durrell Wildlife Conservation Trust, Les Augrés Manor, Trinity, Jersey, JE3 5BP, UK Channel Islands. E-mail richard.young@durrell.org

Anselme Toto Volahy, Robert Bourou, Richard Lewis and Joanna Durbin Durrell Wildlife Conservation Trust, BP8511, Antananarivo 101, Madagascar.

Received 20 October 2006. Revision requested 29 November 2006. Accepted 7 January 2007.

Article updated on 12 August 2022 slash-and-burn clearance for subsistence agriculture, and charcoal production. By 1990 only $2.8 \%$ of the original extent of this habitat was estimated to remain (Smith, 1997), and dry forest is now one of the highest conservation priorities in Madagascar (Ganzhorn et al., 2001). One of the largest remaining areas of dry forest occurs in the central Menabe region and supports a number of locally endemic animal species, including the Madagascar flat-tailed tortoise Pyxis planicauda. This species is now restricted to sparsely populated forest fragments and remains threatened by continuing habitat destruction and possibly by illegal collection for the pet trade, which is thought to have led to localized extinction in a number of small forest patches (J. Durbin, unpubl. data).

P. planicauda is listed on Appendix I of CITES (2008), and is categorized as Endangered on the Red List (IUCN, 2007). The species is vulnerable to overexploitation because of its particularly slow reproductive rate (1-3 eggs per year) and long generation time (maturity at 12-14 years; Glaw \& Vences, 1994). During a Conservation Assessment and Management Plan workshop in Madagascar in 2001 the total population size of $P$. planicauda was estimated to be $<10,000$ individuals, and the extent of occurrence $<5,000 \mathrm{~km}^{2}$, with an area of actual occupancy of $<500 \mathrm{~km}^{2}$ (IUCN/CSBG, 2002). Population surveys of $P$. planicauda in small forest patches (J. Durbin, unpubl. data) and at localized sites in large fragments (Bloxam et al., 1996; Toto Volahy et al., 2002) have been carried out, yet little is known about the abundance and distribution of this species across the majority of its range.

Little is known of the behaviour of this species but $P$. planicauda is known to aestivate through the western Madagascar dry season and increase its activity in the wet season from December to March, when it breeds (Kuchling \& Bloxam, 1988). However, activity patterns are thought to be closely associated with rainfall and individuals reduce their activity during dry periods in the wet season. $P$. planicauda are hard to detect, unless in close proximity, as they are well camouflaged against leaf litter and often hidden in dense forest understorey, and therefore incorporating a method of estimating the probability of detection in any survey of population abundance is vital.

Distance sampling (Buckland et al., 2001) is a widely used survey technique that allows detection probability to 
be estimated and has been used to survey and monitor tortoises elsewhere (Swann et al., 2002; Leuteritz et al., 2005). However, to produce an unbiased density estimate using distance sampling there are a number of assumptions that must be met in the survey design and data collection protocols (Buckland et al., 2001). The most important assumption is that animals on or near the line transect are always detected (i.e. detection probability at zero distance, $g(0)=1)$. This is often problematic when surveying cryptic species where behaviour or habitat structure can prevent animals from being observed on the line, even if present, and can lead to underestimation of density (Buckland et al., 2001; Swann et al., 2002). For example, during their active season, $P$. planicauda are known to spend some time completely hidden under leaf litter and would therefore not be available for sampling (i.e. $g(0)<1$ ). The main objective of this study was to develop a survey technique that allows unbiased estimation of $P$. planicauda abundance. We describe a detailed methodology for a distance sampling survey that includes an independent estimate of $g(0)$. Using this, we report the first population abundance estimates of $P$. planicauda over a large part of its distribution and make recommendations for the design of a monitoring programme.

\section{Study area}

The dry forests of the Menabe region are primarily on sandy and lateritic soils and are characterized by the presence of baobab trees (four species of Adansonia; Rauh, 1995). The climate of this region is dominated by two major seasons: a dry season from April to November, with a wet season in the intervening period. P. planicauda were surveyed within the main central Menabe forest block $(68,976$ ha; Fig. 1), which is thought to represent the vast majority of the species' distribution range. The survey area was divided into two relatively undisturbed northern (Ambadira forest) and southern (Kirindy forest) zones, connected by a more degraded corridor zone (Fig. 1). A 12,500 ha former forest concession in the Kirindy forest, managed as a sustainable logging concession by the Centre de Formation Professionelle Forestière, probably represents the least disturbed area of forest in the region.

\section{Methods}

\section{Line transect survey}

During a previous plot sampling survey of this species in the southern forest zone in 2002, 39 tortoises were observed (Toto Volahy et al., 2002). This fieldwork represented c. 200 $\mathrm{km}$ of line transect survey effort. These data were used to estimate an approximate encounter rate of $P$. planicauda in the central Menabe forest of 0.195 tortoises $\mathrm{km}^{-1}$. Using the equation provided by Buckland et al. (2001) to calculate survey effort, we estimated $384.6 \mathrm{~km}$ of total survey length would be required to achieve a density estimate with a coefficient of variation of $20 \%$. This was considered an acceptable level of precision given the objectives of the survey. The planned survey effort was divided into 49 line transects, each of $3 \mathrm{~km}$ in length, which were positioned randomly throughout the Menabe forest but stratified by survey zone (Fig. 1) so that number of transects allocated to each zone was in proportion to its area. It was planned that line transects would be repeated to achieve the target number of observations (60-80) necessary for modelling detection probability. As tortoises were thought to be most active only during January-February, and the movement of fieldworkers in the region can be seriously inhibited by flooded roads and rivers, the survey was undertaken over two separate wet seasons; 29 transects were surveyed between 16 January and 4 March 2004 and 20 transects between 17 and 29 January 2005. The 2004 survey was interrupted by a major cyclone in the region, during which fieldwork was suspended. Transects were surveyed at 07.0012.00 and 14.00-17.00 but only if rain had fallen in the previous 3 days, after which tortoises are thought to reduce their activity levels.

To maximize efficiency, transects were square in shape with each side $c$. $700 \mathrm{~m}$ long, which allowed surveyors to return to the start point of the transect whilst surveying continuously. The surveyor walked slowly along the line transect, searching for tortoises. A co-worker walked behind the surveyor ensuring a predetermined bearing was followed by means of a compass and to measure the distance walked using a hip chain (Forestry Suppliers, Jackson, USA). The perpendicular distance between the line transect (as marked by the twine from the hip chain) and the central point of the carapace of any tortoises detected, was measured to the nearest $\mathrm{cm}$ using a measuring stick. All tortoises were marked by cutting a series of small notches along the edge of the carapace and examined to determine sex, age and weight before being released.

\section{Estimating $g(0)$}

The proportion of time tortoises spent above leaf litter and therefore available for sampling on the line (i.e. $g(0)$ ) was estimated through repeated observations of a number of individuals $(n=12)$ using spool and line tracking (Boonstra \& Craine, 1985) to relocate animals. Twelve individuals (eight females, three males, one juvenile) were selected randomly from those observed during the 2004 survey and from incidental captures in the same year. A spool and line device, consisting of quilting cocoons of c. $150 \mathrm{~m}$ nylon thread (Danfield, Leigh, UK), was attached to the tortoise's carapace using superglue (Henkel Loctite Adhesives, Hemel Hempstead, UK) and the thread tied to a nearby tree before the animal was released. Tortoises were 


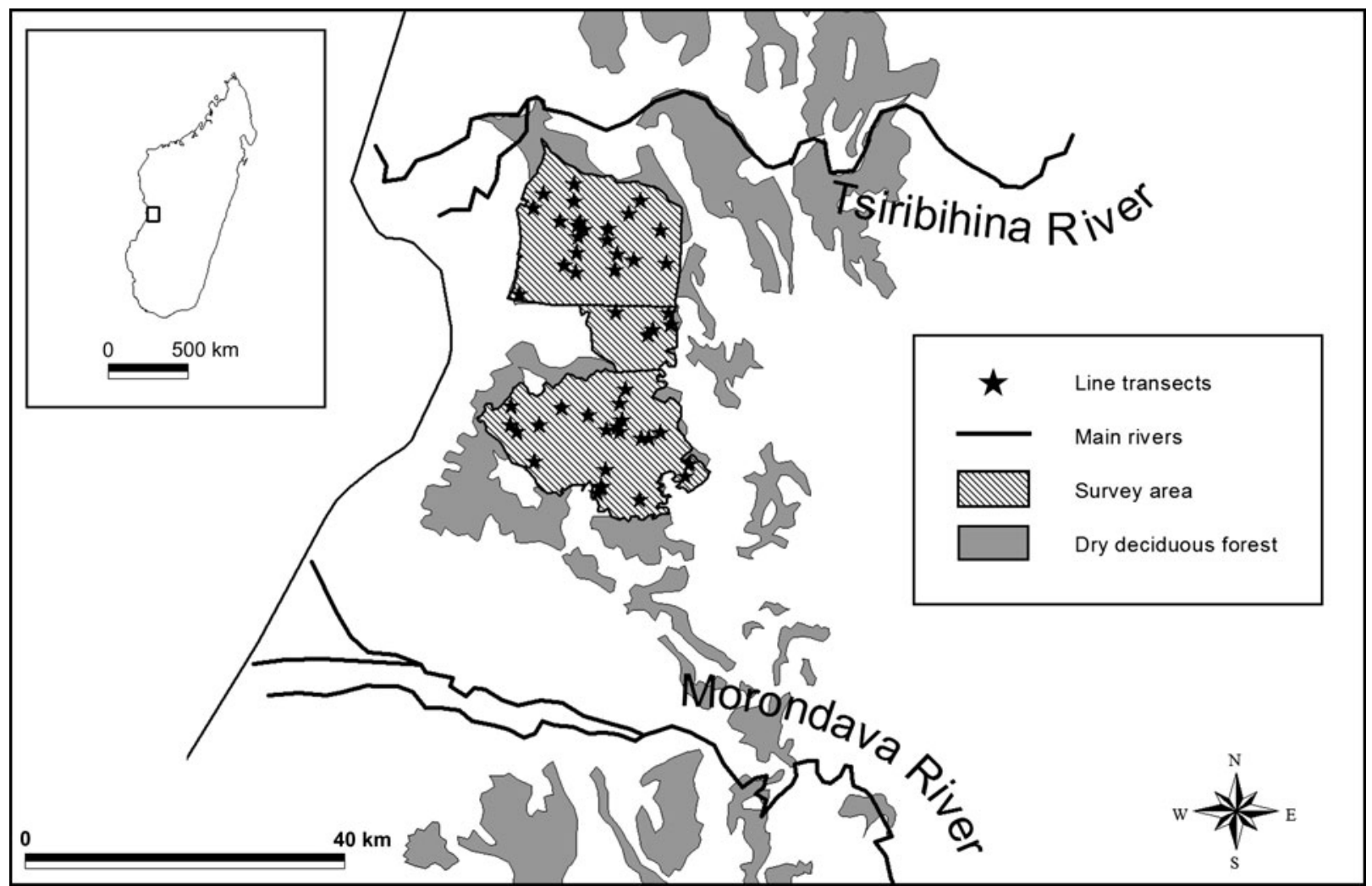

FIG. 1 The distribution of line transects across the northern, central and southern zones of the survey area in the deciduous, dry forests of central Menabe, Madagascar. The inset shows the location of central Menabe within Madagascar.

relocated at hourly intervals during the day, between 07.00 and 18.00 , by following the thread until the tortoise was found. Individuals were classified as being visible (i.e. the whole or some part of the body was above the leaf litter), or hidden (i.e. completely hidden under leaf litter and therefore not available for sampling). However, only relocations that were made during the times of surveying (i.e. 07.0012.00 and 14.00-17.00) and within 3 days of rain were included in the estimate of $g(0)$. The mean proportion of time tortoises were visible was calculated and used as an estimate of $g(0)$ in the distance analysis. A 95\% confidence interval (CI) of $g(0)$ was calculated using non-parametric bootstrapping ( $\mathrm{n}=1,000$ iterations). The distance tortoises moved between relocations was also measured, and divided by the time between relocations to calculate speed of movement and then compared to time of day to investigate daily patterns of activity.

\section{Data analysis}

The perpendicular distance data were examined in a histogram of 12 intervals of equal width to investigate any responsive movement to the observer and clumping of observations. No strong evidence of evasive movement was detected and therefore the data were analysed as ungrouped but truncated so that the $5 \%$ of distances furthest from the line were excluded. Tortoise density was estimated using the software Distance v. 4.0 (Thomas et al., 2003), including the estimate of $g(0)$ and associated standard error. The analysis was post-stratified by year so that density was estimated using a global detection function but encounter rate was estimated separately in each year. Overall density for the survey was estimated from the mean of annual estimates, weighted by total survey effort in each year. All models recommended by Buckland et al. (2001) were considered for density estimation. For each the detection probability histogram and goodness of fit statistics were examined. On the basis of a low Akaike's Information Criterion (AIC) and \% coefficient of variation, the uniform key function model with one cosine adjustment was selected to fit the detection function and estimate density (Table 1). An analysis of variance was used to test differences in tortoise activity between time periods, taking into account the effect of repeated sampling of individual animals.

\section{Results}

\section{Tortoise detectability and movement patterns}

Tortoises were tracked for an average of $5.13 \pm$ SE 0.83 days and a mean of $29.92 \pm \mathrm{SE} 3.58$ relocations were made per individual. The mean proportion of time tortoises were detectable, i.e. $g(0)$, was $0.940 \pm$ SE 0.023 (95\% CI $0.89-0.98$ ). 
TABLE 1 Mean density, with coefficient of variation (CV), and abundance estimates, with lower and upper 95\% confidence intervals (CI), of Pyxis planicauda in the main forest block of central Menabe during 2004-2005.

\begin{tabular}{|c|c|c|c|c|c|c|}
\hline Model & $\Delta \mathrm{AIC}^{*}$ & $\begin{array}{l}\text { Density } \\
\left(\text { tortoises } \mathrm{ha}^{-1} \text { ) }\right.\end{array}$ & CV (\%) & Abundance & Lower 95\% CI & Upper 95\% CI \\
\hline Half normal & 0.00 & 0.405 & 29.6 & 27,921 & 15,682 & 49,712 \\
\hline Uniform cosine & 0.09 & 0.404 & 27.3 & 27,894 & 16,323 & 47,669 \\
\hline Uniform simple polynomial & 0.35 & 0.353 & 26.3 & 24,318 & 14,484 & 40,828 \\
\hline Hazard rate & 2.47 & 0.355 & 30.7 & 24,488 & 13,462 & 44,543 \\
\hline
\end{tabular}

*Akaike's Information Criterion

Patterns of tortoise activity varied during the day (ANOVA $F_{4,44}=3.33, \mathrm{P}=0.018$ ) with a peak in movement over 09.30-11.30 (Fig. 2). Rates of movement were low and even during the most active period tortoises only moved at a mean speed of $4.9 \mathrm{~m} \mathrm{hr}^{-1}$.

\section{Tortoise density}

Because of unfavourably dry conditions during the wet seasons in 2004 and 2005, the time considered suitable for surveying was limited and consequently it was only possible to survey each of the 49 line transects once, which gave a total survey effort of $107.9 \mathrm{~km}$. A total of 29 tortoises were observed, fewer than the 60-80 individuals recommended for modelling the detection function. However, the analysis ran without error in Distance and all models produced similar estimates of density (Table 1). The uniform model with one cosine adjustment provided a good fit of the data $\left(\chi^{2}=2.74, \mathrm{df}=4, \mathrm{P}=0.60\right)$, and was used to estimate density because the AIC value was only marginally different to the half-normal models but was of greater precision (Table 1).

Including the estimate of $g(0)$ in the analysis, mean density of $P$. planicauda was estimated to be 0.404 individuals ha ${ }^{-1}$ (95\% CI $0.237-0.691$ ). Extrapolating from the area sampled, the total population size of $P$. planicauda was estimated to be 27,894 individuals in the main central Menabe forest block (95\% CI 16,323-47,669). The \% coefficient of variation was $27.3 \%$ and although this is higher than the targeted level of precision (20\%), it is relatively low considering the small number of individuals detected in the survey and the incorporation of an estimate of $g(0)$. This uncertainty is reflected in the wide confidence interval abundance estimate and came from three sources: detection probability (2004, 9.3\%; 2005, 10.2\%), encounter rate (2004, $90.3 \%$; 2005, 89.4\%) and $g(0)(2004,0.4 \%$; 2005, 0.5\%).

The probability of detecting tortoises, $g(\mathrm{y})$, fell quickly with increasing distance from the line (Fig. 3). This is likely to be because of a combination of the cryptic nature of the species and the influence of the typically dense understorey vegetation during the wet season. The detection function had a narrow effective strip half-width of $3.41 \mathrm{~m}$ and revealed that at $6 \mathrm{~m}$ from the line there was $<20 \%$ probability of detecting a tortoise when present (Fig. 3).

\section{Discussion}

Despite only 29 observations it was possible to produce a density estimate of reasonable precision, leading to a point estimate of $P$. planicauda population size of nearly 28,000 individuals for the main central Menabe forest block. As the survey covered the vast majority of the species' range,

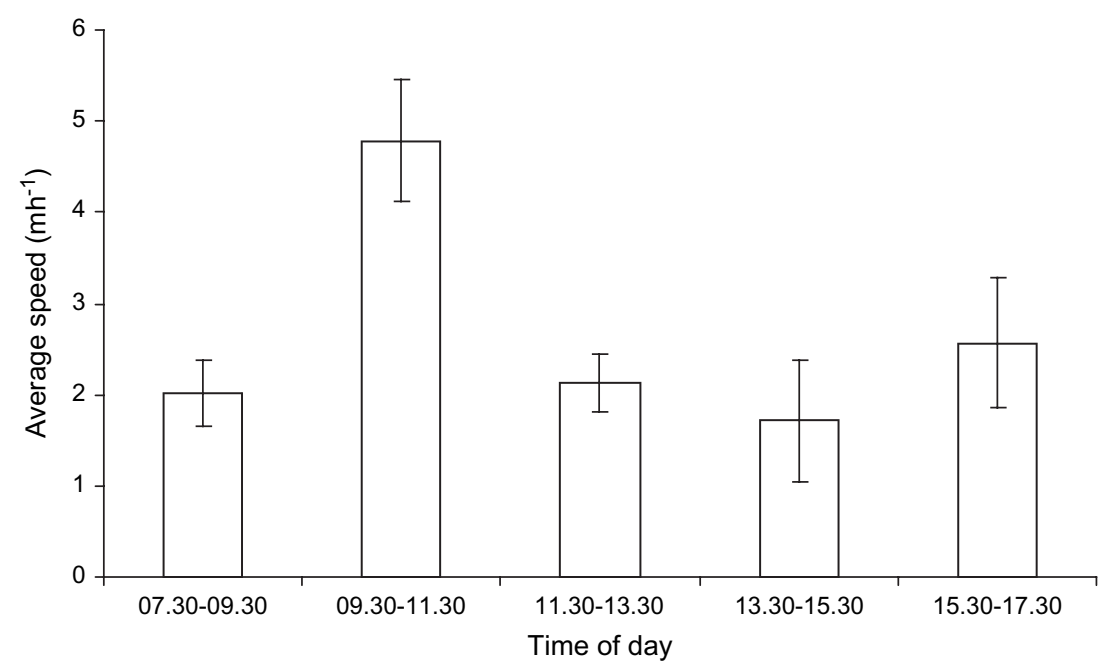

FIG. 2 Daily activity patterns, as average speed, of Pyxis planicauda. Bars are standard errors. 


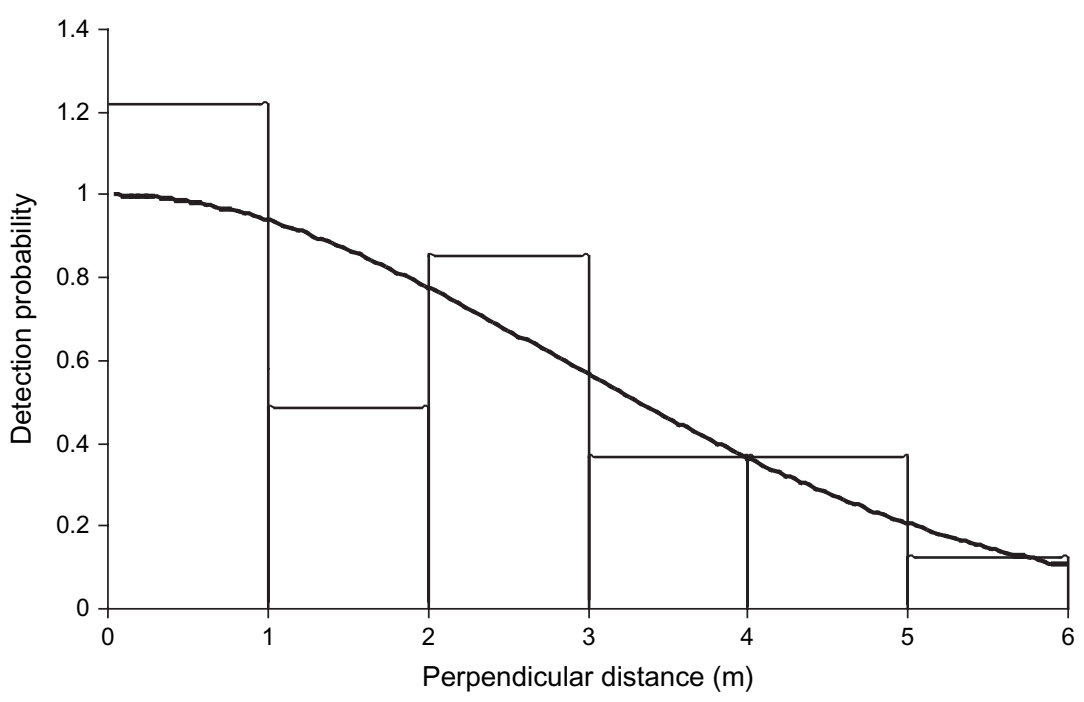

FIg. 3 Detection probability $g(y)$ of Pyxis planicauda in central Menabe during 20042005. except a small number of isolated forest fragments, this represents the most reliable estimate of population size of $P$. planicauda to date. However, because of uncertainty in the density estimate the confidence interval estimate of abundance was relatively wide. Population size was greater than previously assumed and is substantially higher than the earlier prediction of $<10,000$ individuals (IUCN/CSBG, 2002). In light of these results the threatened status of $P$. planicauda may warrant re-evaluation, although this process would need to consider deforestation rates and the resulting probable change in extent of species occurrence, which is beyond the scope of this study.

$P$. planicauda has been estimated to occur at a density of 2-6 individuals $\mathrm{ha}^{-1}$ in highly degraded small forest fragments (J. Durbin, unpubl. data) and 5.9 tortoises $\mathrm{ha}^{-1}$ in one localized 2 ha site in the Kirindy forest (Bloxam et al., 1996). However, the latter estimate was probably an overestimate because of the long sampling period used. The mean density of 0.40 tortoises $\mathrm{ha}^{-1}$ in our study is much lower than these figures but considering our robust survey design we believe it to be representative of the wider population. It is unclear whether this density is near carrying capacity for a tortoise species with such an unusual life history, or whether illegal collection has had a major impact on numbers of $P$. planicauda. In comparison, despite illegal collection activities, the larger bodied radiated tortoise Geochelone radiata in xeric forest in southern Madagascar occurs at densities of $c .25$ tortoises ha ${ }^{-1}$ (O'Brien et al., 2003; Leuteritz et al., 2005).

In our study it was not possible to analyse spatial variations in abundance or habitat associations of P. planicauda because of the relatively low number of observations. More survey data are needed to identify and map areas that support abundant populations so that they can be managed for conservation. The main Menabe forest block represents the majority of the species' range, and therefore the conservation of this area is vital to the long-term survival of
P. planicauda, as well as other vertebrate species endemic to the region (e.g. Microcebus berthae, Hypogeomys antimena, Mungotictis decemlineata decemlineata and Aglyptodactylus laticeps).

A protected area that encompasses the central Menabe forest has recently been designated by the Government of Madagascar, and will be managed to conserve biodiversity and provide incentives for the sustainable use of forest resources by local people. An important element of the management plan for the protected area will be the implementation of monitoring programmes for regionally endemic and endangered species, including $P$. planicauda, to enable the effectiveness of the conservation action to be quantified. This study has provided a survey protocol and baseline abundance estimate with which to monitor future changes in the population size of $P$. planicauda.

Distance sampling is an effective survey technique for monitoring P. planicauda. However, because of the low population density and cryptic nature of this species, and the logistical difficulties of conducting fieldwork in the Menabe region in the wet season, it will be difficult to achieve $60-80$ observations (the recommended sample size for distance sampling) in a given year with the resources likely to be available. Nevertheless, despite far fewer observations, and the inclusion of an estimate of $g(0)$, the relatively low \% coefficient of variation in the density estimate reported here suggests it will not be necessary to achieve this sample size to produce a density estimate of acceptable precision. Small numbers of observations also result in less information with which to judge whether the modelled detection function is a reasonable approximation of how detection probability of tortoises declines with distance. If standardized data collection protocols are used (and assuming no large changes in habitat structure), population abundance can be estimated annually with greater precision and reliability by pooling data across different 
years to model the detection function but post-stratifying density estimation by year.

Given that behaviour can have a profound effect on the accuracy of density estimation it is vital that an independent estimate of $g(0)$ is incorporated into a future monitoring programme (Swann et al., 2002). The activity of $P$. planicauda is probably closely correlated with the amount of rainfall and hence $g(0)$ is likely to vary between years according to differing rainfall patterns. Therefore a reliable estimate of $g(0)$ will be needed for each year or sampling session. For example, we only estimated $g(0)$ in 2004 and if behaviour changed dramatically in 2005 as a result of different rainfall patterns bias may have been introduced into the global density estimate. However, as we kept the data collection protocol consistent in both years of the survey, and only surveyed during periods of rain and for 3 days after the last rain, we believe any bias to be minimal. Our sample was skewed towards females, although it was broadly representative of those animals caught during the survey (14 females, nine males and six juveniles). It is not clear whether this female-biased sex ratio reflects the wider population in the region. Data from future surveys will allow this to be investigated further.

The mean proportion of time that tortoises were visible, and therefore available for sampling, was higher than expected at $94.0 \%$. This may be because we monitored the detectability of tortoises during the time they are presumed to be most active (i.e. during and shortly after rain). The longest period of time an individual monitored tortoise was continuously hidden under leaf litter was 28 hours. However, our sample of tortoises may be biased against individuals that spend substantial periods of time hidden under leaf litter as it was only possible to capture visible animals, which are likely to be the most active. If this is the case $g(0)$ may have been overestimated, and consequently population size underestimated. Clearly, further investigation of the activity patterns of $P$. planicauda is needed, particularly the effect of rain on $g(0)$ and therefore on the reliability of population trend estimation. Despite these issues, spool and line tracking proved to be a cheap and efficient technique for estimating $g(0)$.

Patterns of activity of $P$. planicauda varied during the day with a peak between 09.30 and 11.30, which is probably when tortoises are most easily detected and therefore the optimal time for surveying. However, restricting sampling to such a short time period would inevitably reduce the number of line transects that can be surveyed and therefore the number of observations for density estimation. We recommend that data collection during future surveys should be from 07.00 to 12.00 , which encompasses the most active period of $P$. planicauda but would not have a major impact on the efficiency of the survey.

The recent declaration by the Madagascar Government of a protected area for central Menabe provides hope that the majority of the remaining dry forest habitat in this region will be protected in the long term. The survival of P. planicauda, however, is likely to remain threatened by illegal collection. Export permits issued by the Madagascar Government amounted to 1,367 individuals in 2000 and 534 in 2001 and import figures to other countries indicated that the actual numbers exported were likely to be even higher (R. Reeve, unpubl. data). The species was added to Appendix I of CITES in 2001 but we believe that the threat of illegal trade persists because in December 2005 local people in Menabe were contacted by a collector seeking to purchase P. planicauda (W. Rakotombololona, pers. comm.). Given its low fecundity and long generation time, any offtake, particularly of reproductive adults, is likely to pose a significant threat to this species.

\section{Acknowledgements}

We are grateful to Conservation International and the United States Agency for International Development for funding this survey and our project partners Fanamby for their support. We thank the villagers of Beroboka, the Deutsches Primatenzentrum and Centre de Formation Professionelle Forestière for logistical assistance. We thank Jonah Ratsimbazafy, William Rakotombololona, Tim Hounsome and Quentin Bloxam for their valuable contributions to the project. Two anonymous reviewers provided useful comments on an earlier draft.

\section{References}

Bloxam, Q.M.C., Nody, J.P., Rabenjanahary, R.D. \& Gibson, R. (1996) Estimating density and abundance of the Madagascar flat-tailed tortoise. Dodo, 32, 132-136.

Boonstra, R. \& Craine, I.T.M. (1985) Natal nest location and small mammal tracking with a spool and line technique. Canadian Journal of Zoology, 64, 1034-1036.

Buckland, S.T., Anderson, D.R., Burnham, K.P., LaAke, J.L., Borchers, D.L. \& Thomas, L. (2001) Introduction to Distance Sampling: Estimating Abundance of Biological Populations. Oxford University Press, Oxford, UK.

CITES (2008) The CITES Appendices. Http://www.cites.org/eng/app/ index.shtml [accessed 29 January 2008].

Ganzhorn, J.U., Lowry II, P.P., Schatz, G.E. \& Sommer, S. (2001) The biodiversity of Madagascar: one of the world's hottest hotspots on its way out. Oryx, 35, 346-348.

Glaw, F. \& Vences, M. (1994) A Field Guide to the Amphibians and Reptiles of Madagascar, 2nd edition. Vences \& Glaw Verlag, Köln, Germany.

Guillaumet, J.J. (1984) The vegetation: an extraordinary diversity. In Key Environments: Madagascar (eds A. Jolly, P. Oberlé \& R. Albignac), pp. 27-54. Pergamon Press, New York, USA.

IUCN (2007) 2007 IUCN Red List of Threatened Species. IUCN, Gland, Switzerland. Http://www.redlist.org [accessed 10 September 2007].

IUCN/CSBG (2002) Evaluation et Plans de Gestion pour la Conservation (CAMP) de la Faune de Madagascar. Lémuriens, Autres Mammifères, Reptiles et Amphibiens, Poissons d'eau douce et Evaluation de la Viabilité des Populations et des Habitats de 
Hypogeomys anitmena (Vositse). Conservation Breeding Specialist Group, Apple Valley, USA.

Kuchling, G. \& Bloxam, Q.M.C. (1988) Field data on the Madagascan flat tailed tortoise Pyxis (Acinixys) planicauda. Amphibia-Reptilia, 9, 175-180.

Leuteritz, T.E.J., Lamb, T. \& Limberaza, J.C. (2005) Distribution, status, and conservation of radiated tortoises (Geochelone radiata) in Madagascar. Biological Conservation, 124, 451-461.

Lowry II, P.P., Schatz, G.E. \& Phillipson, P.B. (1997) The classification of natural and anthropogenic vegetation in Madagascar. In Natural Change and Human Impact in Madagascar (eds S.M. Goodman \& B.D. Patterson), pp. 93-123. Smithsonian Institution Press, Washington, DC, USA

O'Brien, S., Emahalala, E.R., Beard, V., Rakotondrainy, R.M., Reid, A., Raharisoa, V. \& Coulson, T. (2003) Decline of the Madagascar radiated tortoise Geochelone radiata due to overexploitation. Oryx, 37, 338-343.

RAun, W. (1995) Succulent and Xerophytic Plants of Madagascar, Vol I. Strawberry Press, Mill Valley, USA.

Smith, A.P. (1997) Deforestation, fragmentation, and reserve design in western Madagascar. In Tropical Forest Remnants: Ecology, Management and Conservation of Fragmented Communities (eds W. Laurance \& O.W. Bierregaard), pp. 415-441. The University of Chicago Press, Chicago, USA.

Swann, D.E., Averill-Murray, R.C. \& Schwalbe, C.R. (2002) Distance sampling for Sonoran desert tortoises. Journal of Wildlife Management, 66, 969-975.
Thomas, L., Laake, J.L., Strindberg, S., Marques, F.F.C., Buckland, S.T., Borchers, D.L. et al. (2003) Distance 4.0 Release 1. Research Unit for Wildlife Population Assessment, University of St. Andrews, UK. Http://www.ruwpa.st-and.ac.uk/ distance/ [accessed 20 March 2005].

Toto Volahy, A., Durbin, J.D. \& Rakotombolona, W.F. (2002) Density and Population Estimates of the Flat Tailed Tortoise Pyxis planicauda in the Western Dry Forest of Madagascar. Unpublished Report. Durrell Wildlife Conservation Trust, Trinity, UK.

\section{Biographical sketches}

Richard Young specializes in animal abundance estimation, and currently works on projects in Madagascar and the Caribbean researching and monitoring threatened species. Anselme Tото Volahy and Robert Bourou are working on a range of threatened species monitoring programmes in western Madagascar. RICHARD LEWIS has conducted research on the ecology and conservation of a number of Malagasy tortoise species and is Conservation Coordinator for the Durrell Wildlife Conservation Trust in Madagascar. JoAnna DuRbin has broad research interests in biodiversity conservation and is the former Programme Director for the Durrell Wildlife Conservation Trust in Madagascar. JULIA FA has undertaken research in a range of conservation biology topics in Europe, Africa and South America, and specializes in biodiversity assessment and threatened species management. 